\title{
Diagnostic and therapeutic aspects in patients with chronic osteomyelitis and prosthetic infections
}

\author{
Vanessa Mary Crapanzano Minichello', Daniela Segala', Lorenzo Badia', Rosario Cultrera', \\ Anastasio Grilli', Leo Massari', Carlo Contini' \\ 'Sezione di Malattie Infettive, Dipartimento di Medicina Clinica e Sperimentale; via Fossato di Mortara, 23, 44 I 00 Ferrara; \\ ${ }^{2}$ Istituto di Clinica Ortopedica, Corso Giovecca, Università di Ferrara
}

Key words: Osteomyelitis, prosthetic joint infections, biofilm, antibiotics, S. aureus, hyperbaric therapy

Aspetti diagnostici e terapeutici in pazienti con osteomieliti croniche ed infezioni protesiche

\section{SUMMARY}

Management of chronic osteomyelitis and prosthetic joint infections represents an emergent problem, despite the advances in antibiotics and new operative techniques. In order to evaluate the efficacy of a multidisciplinary approach we conducted a study on twelve patients with orthopedic devices related infections attending the Institute of Infectious Diseases of the University of Ferrara from 200 I to 2006, by analyzing clinical, laboratoristic and strumental outcomes. The best results were obtained for those patients who underwent surgical treatment, including bone debridement or removal of infected devices, associated with an adequate antibiotic treatment. Moreover, encouraging results were found by analyzing the outcomes from 2 patients who underwent hyperbaric ( $\mathrm{HBO}$ ) and antibiotic therapy, as well as in patients with prosthetic-joint infections treated with antibiotics only. Our experience allows us to confirm the importance of a close interaction between infectious disease specialists, orthopaedic surgeons, radiologists, plastic surgeons, microbiologists and specialists in hyperbaric medicine in the management of bone and prosthetic joint infections.

Received September I2, 2007

Accepted November 5, 2007

\section{INTRODUZIONE}

Nonostante i progressi in campo profilattico e terapeutico, le infezioni osteo-articolari e protesiche rappresentano un problema grave e complesso per l'infettivologo ed il chirurgo ortopedico. Infatti, gli elevati tassi di successo della terapia antibiotica, che si osservano nella maggior parte delle malattie infettive, non sono stati ancora raggiunti nelle infezioni osteoarticolari, a causa delle caratteristiche anatomiche e fisiologiche dell'osso che richiedono trattamenti antibiotici protratti e spesso associati ad interventi chirurgici non sempre realizzabili o risolutivi. Inoltre, con l'incremento della popolazione anziana e dell'impiego sempre più esteso di impianti protesici ortopedici, l'incidenza di queste patologie è destinata ad aumentare.

In Italia vengono ogni anno impiantate circa 50.000 protesi d'anca e 10.000 protesi di ginocchio. Mentre nel 1999 l'incidenza di infezione nelle protesi d'anca si attestava attorno a $1-1.5 \%$ all'anno, con l'aumento dei reimpianti, l'incidenza è allo stato attuale triplicata (3-5\% all'anno). Si può pertanto stimare che in 100 pazienti operati si svilupperanno complessivamente $10-15$ episodi infettivi importanti nell'arco di 10 anni (5).

L'osteomielite cronica è caratterizzata da un'infezione persistente e recidivante che evolve in mesi $\mathrm{o}$ anni ed è caratterizzata, rispetto alla forma acuta, dalla presenza di un minor grado di infiammazione, sequestri, deposizione di tessuto osseo di neogenesi e tratti fistolosi.

Gli schemi classificativi più utilizzati sono quelli di Waldvogel et al. e di Cierny et al. fondati, rispettivamente, sulla patogenesi e sull'anatomia dell'infezione e fisiologia dell'ospite $(5,14,19)$.

Se lo Stafilococco aureo rappresenta uno degli agenti etiologici maggiormente implicati nelle infezioni osteo-articolari, gli stafilococchi coagulasi-negativi, quali $S$. epidermidis, si riscontrano più frequentemente in quelle di protesi; streptococchi, anaerobi, enterococchi, batteri Gram-negativi e miceti si associano invece ad infezioni che coinvolgono principalmente l'immunocompromesso ed il trapiantato. In Italia, inoltre, come conseguenza dei flussi migratori dall'Africa e dall'Asia, si sta assistendo ad un incremento delle osteomieliti vertebrali sostenute da Mycobacterium tuberculosis (8,

\section{Corresponding author: Carlo Contini}

Sezione di Malattie Infettive, Dipartimento di Medicina Clinica e Sperimentale - Università di Ferrara

Via Fossato di Mortara, 23 - 44100 Ferrara - Tel.: 0532455490 - Fax: 0532237707 
14, 18). La diagnosi di osteomielite si avvale oltre che di dati anamnestici ed obiettivi, di esami di laboratorio, strumentali e colturali.

Il razionale per una strategia terapeutica efficace è rappresentato dalla diagnosi precoce, che consiste nell'isolamento microbiologico del germe responsabile e sull'istituzione di un trattamento antibiotico mirato e prolungato. In assenza di una diagnosi eziologica, la terapia medica deve tener conto della realtà epidemiologica locale, della patogenesi e dell'eventuale presenza di dispositivi ortopedici o di protesi (16). Per questi ultimi, in particolare, è doveroso considerare la formazione del "biofilm", ritenuto, secondo i CDC, la causa del $65 \%$ delle infezioni ospedaliere diagnosticate nei paesi avanzati e la produzione di "slime", matrice esopolisaccaridica, capace di mediare le fasi finali della colonizzazione microbica e considerato un importante fattore di virulenza (7).

La profilassi antibiotica costituisce pertanto un obiettivo primario e si articola fondamentalmente sull'utilizzo di cementi antibiotati, su appropriate misure di controllo delle infezioni e su una attenta tecnica chirurgica $(2,6,7,9,11,15,21)$. Recenti studi dimostrano che la terapia antibiotica, protratta per almeno 4-6 settimane, associata ad un accurato "debridement" rappresenti l'approccio ottimale nel management delle infezioni osteoarticolari, in presenza o meno di mezzi di sintesi o protesi, i quali possono essere addirittura rimossi, con o senza reimpianto $(13,20,24)$.

Non numerosi sono gli studi inerenti l'efficacia dell'ossigenoterapia iperbarica (OTI) in corso di osteomielite cronica. Tuttavia, dai risultati pubblicati in letteratura, emerge che l'OTI è efficace quando combinata con una terapia antibiotica e chirurgica adeguate, e l'incidenza di complicanze è relativamente bassa $(1.83 \%$ secondo recenti casistiche). In generale, tale approccio è riservato al trattamento degli stadi avanzati di osteomielite $(3 \mathrm{~B}$ e $4 \mathrm{~B}$, secondo la classificazione di Cierny et al.) $(1,3,4,12,23)$.

Scopo del nostro studio è stato quello di valutare l'adeguatezza dell'approccio infettivologico e chirurgico-ortopedico nel management delle osteomieliti da inoculazione diretta (fratture esposte, contaminazione batterica in seguito ad interventi chirurgici sull'osso), in presenza o meno di mezzi di sintesi e di infezioni protesiche. A tal fine sono stati valutati gli "outcomes" (clinici, laboratoristici e strumentali) relativi a 12 pazienti seguiti presso 1'Istituto di Malattie Infettive dell'Università di Ferrara dal 2001 al 2006 ed ottenuti con l'associazione della terapia antibiotica e/o terapia chirurgica e/o iperbarica.

\section{PAZIENTI E METODI}

In tabella 1 sono esposte le caratteristiche demografiche e cliniche relative ai 12 pazienti ( 6 uomini e 6 donne, gruppi 1-3) seguiti presso il Day Hospital dell'Istituto di Malattie Infettive dell'Università di Ferrara tra il 2001 ed il 2006, affetti da osteomielite cronica (OC). Sono stati suddivisi in 3 gruppi: gruppo 1, pazienti con OC esenti da dispositivi ortopedici; n. 2 casi; gruppo 2, pazienti con OC e mezzi di sintesi (OMS), 7 casi; gruppo 3, pazienti con protesi articolare (OP), 2 casi.

Nella tabella 2 sono esposti la sede e la durata dell'infezione di ciascun paziente, i precedenti isolamenti e le terapie antibiotiche effettuate nel periodo antecedente il ricovero. La diagnosi di infezione è stata effettuata utilizzando criteri clinici generali (dolore, edema, calor, rubor, fistole cutanee, febbre), laboratoristici (conta dei leucociti, VES e PCR), strumentali ( $R x$, ecografia, TAC, RMN, scintigrafia ossea con leucociti marcati) e microbiologici (esame colturale da frammenti ossei, dispositivi ortopedici, materiale da fistola cutanea).

La terapia antibiotica, ove possibile, è stata impostata sulla base dell'isolamento microbiologico (materiale proveniente da fistola o prelevato tramite ago-aspirato o intervento chirurgico); nei restanti casi, la scelta dell'antibioticoterapia si è basata sulla realtà epidemiologica locale e sulle caratteristiche individuali di ciascun paziente.

Sulla scorta dei risultati raggiunti sono stati distinti 3 quadri principali: "risoluzione", normalità del quadro clinico e degli esami ematochimici dopo sei mesi dalla fine della terapia; "miglioramento", assenza di segni clinici d'infezione al termine dei sei mesi dal trattamento, in presenza degli indici di flogosi alterati; "fallimento", segni clinici e strumentali indicativi d'infezione in atto, nonostante la terapia.

\section{RISULTATI}

I pazienti inclusi nel presente studio avevano un'età media pari a 52.6 anni (range 33-76). Tutti i pazienti sono stati monitorati mediante esami ematochimici (compresi indici di flogosi), clinici e strumentali; il periodo medio di follow-up è stato di 18.5 mesi (range 8-66).

Prima del ricovero nel nostro Day Hospital, tutti i pazienti erano stati sottoposti in media a due cicli differenti di terapia antibiotica (tabella 2) con risultati scarsamente soddisfacenti. Durante il ricovero, in 9 pazienti (tabella 3) l'esame colturale è risultato positivo per $S$. epidermidis meticillino-resistente (MRSE) in un caso (n. 3), S. aureus meticillino-sensibile (MSSA) in 5 (n. 1, 2, 4, 5, 8), S. aureus meticillino-resistente (MRSA) in un 
caso (n. 11), Pseudomonas aeruginosa in 2 casi (n. 4,11$)$.

I 2 pazienti appartenenti al gruppo 1 erano stati trattati per 4 settimane con un glicopeptide (teicoplanina, $400 \mathrm{mg} /$ die, e.v) e, successivamente, con un chinolonico (levofloxacina, $500 \mathrm{mg} /$ die, p.o.) e moxifloxacina (400 mg/die, p.o), in un caso; ciprofloxacina, (500 mg x 2/die, p.o), nell'altro. Uno di essi era stato sottoposto ad un intervento di pulizia chirurgica, mentre il secondo aveva eseguito un ciclo di OTI. Entrambi i pazienti sono andati incontro ad una risoluzione completa del quadro clinico-laboratoristico e strumentale.

Del gruppo 2, 6 pazienti erano stati sottoposti per 4 settimane a terapia con teicoplanina (400 $\mathrm{mg} /$ die, e.v), associata, in 3 casi, ad un secondo antibiotico (levofloxacina, $500 \mathrm{mg} / \mathrm{die}$ p.o) od amoxicillina-clavulanato ( $1 \mathrm{~g}$ x $3 /$ die, p.o); un paziente (n. 12), precedentemente trattato con il glicopeptide, è stato trattato con linezolid (600 mg x 2/die, p.o), per 28 giorni, associato ad OTI, senza però ottenere risoluzione o sostanziale miglioramento del quadro clinico-strumentale. In un altro caso invece, (n. 6), la risoluzione completa dei parametri clinici e strumentali ( $\mathrm{Rx}$, ecografia e scintigrafia ossea) è stata osservata con la sola terapia antibiotica (levofloxacina, $500 \mathrm{mg} \mathrm{x}$ 2/die, p.o e rifampicina, $600 \mathrm{mg} / \mathrm{die}$, p.o., preceduta da teicoplanina, $400 \mathrm{mg} /$ die, e.v).

La terapia chirurgica è stata effettuata in 5 pazienti: intervento di pulizia chirurgica associata ad OTI (n. 1), ripetuti interventi di rimozione di mezzi di sintesi e chirurgia plastica (n. 11), rimozione dei mezzi di sintesi (n. 4, 5, 8). In questi ultimi si è assistito a risoluzione completa del quadro clinico-laboratoristico anche se la documentazione strumentale è stata evidenziata in un solo caso. Il paziente sottoposto a pulizia chirurgica ed OTI, è andato incontro ad un miglioramento del quadro clinico, con normalizzazione degli indici laboratoristici, mentre il quadro strumentale era compatibile con infezione ossea. Solo

Tabella I. Caratteristiche demografiche e cliniche dei 12 pazienti studiati.

\begin{tabular}{|c|c|c|c|c|c|c|}
\hline PAZIENTI & ETA' & SESSO & DIAGNOSI & SEDE INFEZIONE & C. & OTI \\
\hline \multirow[t]{2}{*}{ I } & 60 & $M$ & oms & ARTRODESI TIBIO-TARSICA IN ESITI & sì & sì \\
\hline & & & & DI POLIOMIELITE & & \\
\hline 2 & 50 & $M$ & OC & DIAFISI FEMORALE & no & “ \\
\hline 3 & 39 & $\mathrm{~F}$ & OC & TERZO DISTALE OMERO & sì & no \\
\hline 4 & 49 & $\mathrm{~F}$ & oms & FISSATORE INTERNO TIBIA & sì & “ \\
\hline 5 & 60 & $\mathrm{~F}$ & oms & “ & “ & “ \\
\hline 6 & 42 & $\mathrm{~F}$ & oms & TERZO DISTALE TIBIA-PERONE & no & “ \\
\hline 7 & 76 & $\mathrm{~F}$ & op & PROTESI ANCA & “ & “ \\
\hline 8 & 33 & $M$ & oms & FISSATORE INTERNO TIBIA & sì & “ \\
\hline 9 & 73 & $M$ & op & PROTESI ANCA & no & “ \\
\hline 10 & 70 & $\mathrm{~F}$ & “ & “ & “ & “ \\
\hline I I & 38 & $M$ & oms & TIBIA-PERONE & sì & “ \\
\hline 12 & 41 & $M$ & “ & FEMORE (ACETABOLO) & “ & sì \\
\hline
\end{tabular}

C., intervento chirurgico; OTI, terapia iperbarica (cicli medi di 30 sedute); oms, osteomielite su mezzi di sintesi; oc., osteomielite cronica, insorta in assenza del materiale inerte in quanto già rimosso; op, osteomielite su protesi.

Tabella 2. Isolamenti microbiologici, durata dell'infezione e dei trattamenti effettuati presso altre strutture prima del ricovero.

\begin{tabular}{|c|c|c|c|}
\hline PAZIENTI & MICRORGANISMO & DURATA INFEZIONE (mesi) & ANTIBIOTICI \\
\hline I & S. agalactiae multisensibile & 2 & $\mathrm{Amx} / \mathrm{Clv}^{\mathrm{C}}$ \\
\hline 2 & N.D. & 15 & $C f x^{d}$ \\
\hline 3 & N.D. & 60 & $C f x^{c}$ \\
\hline 4 & S. aureus multisensibile & 17 & $\mathrm{Amx} / \mathrm{Clv}+\mathrm{Clr}^{\mathrm{a}} ; \mathrm{Cfx}^{\mathrm{c}}$ \\
\hline 5 & N.D. & 36 & $\mathrm{Amx} / \mathrm{Clv}^{\mathrm{C}}$ \\
\hline 6 & N. D. & 4 & $\mathrm{Am} \times / \mathrm{Clv}$ e \\
\hline 7 & N. D. & 13 & MER + Lfx ${ }^{c}$ \\
\hline 8 & S. aureus multisensibile & II & $C f x^{c}$ \\
\hline 9 & N. D. & 216 & Mfx + RMP b;Teico d \\
\hline 10 & S. aureus $M R, P$. aeruginosa & 12 & Cfx ${ }^{\text {a: }}$ Teico ${ }^{c}$ \\
\hline I I & N.D. & 12 & Amx/clv + Teico b \\
\hline 12 & S. aureus $M R$ & 35 & $\begin{array}{c}\text { Amx/Clv + Lfx } \\
\text { Teico + IMP d }\end{array}$ \\
\hline
\end{tabular}

S., Staphylococcus; P., Pseudomonas; N.D., non disponibile; abbreviazioni antibiotici: “Amx/Clv”,Amoxicillina/Clavulanato; “Cfx”, Ciprofloxacina; “Clr”, Claritromicina; “MER”, Meropenem; “Lfx”, Levofloxacina; “Mfx” Moxifloxacina; “RMP”, Rifampicina; "Teico”, Teicoplanina; “IMP”, Imipenem-cilastatina; Durata della terapia: “a”, I settimana;”b”, 2 settimane; “c”, 4 settimane;“ $d ”, 8$ settimane;“e”, I 2 settimane. 
Tabella 3. Esami microbiologici e principali chemioterapici utilizzati.

\begin{tabular}{|c|c|c|}
\hline PAZIENTI & MICRORGANISMO & ANTIBIOTICI \\
\hline $\mathbf{I}$ & S. aureus MS & 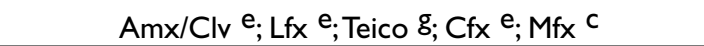 \\
\hline 2 & S. aureus MS & $\mathrm{Amx} / \mathrm{Clv}+\mathrm{Cftr}^{\mathrm{C}} ;$ Teico $^{\mathrm{f}}+\mathrm{RMP}{ }^{\mathrm{b}} ;$ Lfx $^{\mathrm{C}} ;$ Teico $^{\mathrm{f}} ; \mathrm{Mfx}^{\mathrm{c}}$ \\
\hline 3 & S. epidermidis $M R$ & Amx/Clv ${ }^{c} ;$ Teico ${ }^{c} ; \mathrm{Cfx}^{\mathrm{C}}$ \\
\hline 4 & $\begin{array}{l}\text { P. aeruginosa multiresistente; } \\
\text { S. aureus MS }\end{array}$ & IMP + RMP c ;Amx/Clv e;Teico + Lfx c \\
\hline 5 & S. aureus MS & $\mathrm{Lfx}^{\mathrm{C}} ;$ Teico $+\mathrm{Amx} / \mathrm{Clv}^{\mathrm{C}}$ \\
\hline 6 & N.D. & Teico e; Lfx + RMP e \\
\hline 7 & “ & Teico + MER + Lfx C; Cfx + RMP c; Lzd C \\
\hline 8 & S. aureus MS & Teico e; Lfx + RMP c \\
\hline 9 & N.D. & $\mathrm{Amx} / \mathrm{Clv}+\mathrm{RMP} f$ \\
\hline 10 & N.D. & Lzd + IMP d \\
\hline II & $\begin{array}{c}\text { S. aureus MR; } \\
\text { P. aeruginosa multiresistente }\end{array}$ & IMP C; Teico + Lfx e \\
\hline 12 & N.D. & Lfx + Cftr c; Lzd ${ }^{\mathrm{e}} ; \mathrm{Lzd}^{+}+\mathrm{RMP}{ }^{\mathrm{b}}$ \\
\hline
\end{tabular}

S., Staphylococcus; MS, Meticillino-sensibile; MR, Meticillino-resistente; N.D., Non determinato; “Amx/Clv”,'Amoxicillina/Clavulanato; “Lfx”, Levofloxacina; "Teico", Teicoplanina; "Cfx”, Ciprofloxacina; "Mfx", Moxifloxacina; "Cftr”, Ceftriaxone, ; "RMP”, Rifampicina; "IMP”, Imipenem-cilastatina;“MER”, Meropenem;“'Lzd”, Linezolid; Durata della terapia:“a”, I settimana,”b”, 2 settimane, “c”, 4 settimane, “d”, 7 settimane, “e”, 8 settimane, "f”, 12 settimane, "g", 16 settimane.

Tabella 4. Periodi di follow-up e outcomes clinici, laboratoristici e strumentali

\begin{tabular}{|c|c|c|c|c|c|c|c|c|c|}
\hline \multirow{3}{*}{ PAZIENTI } & \multirow{3}{*}{$\begin{array}{l}\text { FOLLOW-UP } \\
\text { (mesi) }\end{array}$} & \multicolumn{8}{|c|}{ OUTCOMES } \\
\hline & & \multirow{2}{*}{ CLINICI } & \multicolumn{3}{|c|}{ LABORATORISTICI } & \multicolumn{4}{|c|}{ STRUMENTALI } \\
\hline & & & LEUCOCITI & VES & PCR & $\mathbf{R X}$ & ECO & $\mathbf{R M N}$ & SCINT. \\
\hline I & 18 & B & $\mathrm{N}$ & $\mathrm{N}$ & $\mathrm{N}$ & + & N.D. & + & + \\
\hline 2 & 15 & $\mathrm{~A}$ & $\mathrm{~N}$ & $\mathrm{~N}$ & $\mathrm{~N}$ & - & - & - & - \\
\hline 3 & 18 & $A$ & $\mathrm{~N}$ & $\mathrm{~N}$ & $\mathrm{~N}$ & N.D. & - & N.D. & N.D. \\
\hline 4 & 12 & $\mathrm{~A}$ & $\mathrm{~N}$ & $\mathrm{~N}$ & $\mathrm{~N}$ & N.D. & N.D. & N.D & N.D \\
\hline 5 & 22 & $A$ & $\mathrm{~N}$ & $\mathrm{~N}$ & $\mathrm{~N}$ & N.D. & N.D. & N.D & N.D \\
\hline 6 & 8 & $\mathrm{~A}$ & $\mathrm{~N}$ & $\mathrm{~N}$ & $\mathrm{~N}$ & - & - & N.D. & - \\
\hline 7 & 11 & $\mathrm{~B}$ & $\uparrow$ & $\uparrow$ & $\uparrow$ & + & + & N.D. & + \\
\hline 8 & 13 & A & $\mathrm{N}$ & $\mathrm{N}$ & $\mathrm{N}$ & - & - & - & - \\
\hline 9 & 9 & B & $\uparrow$ & $\uparrow$ & $\uparrow$ & N.D. & N.D. & N.D. & + \\
\hline 10 & 20 & B & $\uparrow$ & $\uparrow$ & $\uparrow$ & + & + & N. D. & + \\
\hline II & 66 & C & $\uparrow$ & $\uparrow$ & $\uparrow$ & + & + & + & + \\
\hline 12 & 10 & C & $\uparrow$ & $\uparrow$ & $\uparrow$ & N.D. & + & N.D. & + \\
\hline
\end{tabular}

Outcomes clinici:A, Risoluzione completa; B, Miglioramento; C, Fallimento. Outcomes strumentali: N. D., Non disponibile; SCINT.: Scintigrafia ossea con leucociti marcati.

un paziente pluritrattato chirurgicamente (n. 11) ha subito l'amputazione dell'arto.

Dei pazienti del gruppo 3 precedentemente trattati con altri antibiotici inclusi la teicoplanina, due (n. 7, 10) hanno effettuato terapia con linezolid (600 mg x 2/die, p.o), per 28 giorni, associata, in 1 caso ad imipenem (500 mg x 3/die, e.v). In essi si è assistito ad un miglioramento dei sintomi e dei segni clinici, con ripresa della funzionalità motoria e chiusura del tramite fistoloso, ove presente. Nel terzo paziente (n. 9) il miglioramento clinico è stato raggiunto con l'associazione amoxicillina-clavulanato $(1 \mathrm{~g} \mathrm{x} 3 /$ die, p.o) e rifampicina $(600 \mathrm{mg} / \mathrm{die}$, p.o), nonostante la persistenza degli indici di flogosi elevati ed un quadro strumentale compatibile con infezione periprotesica.

\section{DISCUSSIONE}

Le osteomieliti su mezzi di sintesi e su protesi sono infezioni gravi in cui i tempi e le modalità di trattamento antibiotico non sono completamente standardizzati. La maggior parte degli studi dimostra che 4-6 settimane di terapia antibiotica associata ad un accurato debridement e/o rimozione di mezzi di sintesi o protesi possa permettere l'eradicazione dell'infezione.

Tuttavia la scelta della strategia terapeutica deve basarsi sull'attenta valutazione di ogni singolo paziente, sull'isolamento microbiologico e sulla presenza o meno di dispositivi ortopedici $(10,17,22)$.

Nel nostro studio i pazienti erano già stati sottoposti a differenti schemi terapeutici presso altri 
centri con rimozione, in due casi, dei mezzi di sintesi.

Nei pazienti sottoposti ad intervento chirurgico di pulizia (un caso) o di rimozione dei mezzi di sintesi (tre casi), in associazione a terapia con il glicopeptide proseguita fino ad almeno un mese dopo l'intervento, sono stati riscontrati i risultati clinico-laboratoristici più soddisfacenti, oltre ad una normalizzazione del quadro strumentale (2 casi). Infine, il paziente sottoposto a ripetuti interventi di rimozione dei mezzi di sintesi e chirurgia plastica ricostruttiva, per l'interessamento di più segmenti e la particolare gravità del quadro clinico, è andato incontro ad amputazione dell'arto. La limitata casistica analizzata non ci consente di trarre conclusioni definitive sull'utilità dell'OTI nella gestione delle infezioni ossee. Nel nostro studio, 2 pazienti hanno presentato un risultato soddisfacente associando l'OTI con un'adeguata terapia antibiotica e/o "debridement". In un caso, tuttavia, gli esiti di una pregressa infezione poliomielitica ne hanno condizionato significativamente l'efficacia anche dopo cicli ripetuti.

Relativamente alle infezioni di protesi, in accordo con quanto riportato in letteratura, il trattamento chirurgico è quello di scelta, anche se non sempre è possibile realizzarlo, considerata l'età avanzata dei pazienti e l'elevato rischio conseguente ad un espianto. Nel nostro studio, i 3 pazienti appartenenti a questo gruppo, pur avendo effettuato il solo trattamento antibiotico, hanno presentato un netto miglioramento del quadro clinico.

I dati ottenuti dalla nostra casistica dimostrano come la terapia antibiotica associata alla chirurgia rappresenti il migliore approccio nella gestione delle osteomieliti croniche su mezzi di sintesi. Per quanto riguarda le osteomieliti su protesi occorre considerare i diversi fattori che controindicano l'intervento chirurgico ed in particolare l'età avanzata del paziente. Dai dati finora emersi, il trattamento delle infezioni osteoarticolari e protesiche deve contemplare un approccio multidisciplinare, che coinvolge infettivologi, ortopedici, radiologi, chirurghi plastici e specialisti in medicina iperbarica.

La scelta del trattamento antibiotico è assai difficile, e se non sostenuta dall'isolamento microbiologico, deve tener conto della realtà epidemiologica locale, della patogenesi, dell'eventuale presenza di dispositivi ortopedici o protesici e delle condizioni dell'ospite. Pertanto, l'apporto microbiologico diventa determinante nei casi di isolamento di microrganismi difficili o multiresistenti in soggetti sottoposti a precedenti cicli di terapia antibiotica ed in quelli con età avanzata.

\section{BIBLIOGRAFIA}

1. Calhoun JH, Cobos JA, Mader JT. Does hyperbaric oxygen have a place in the treatment of osteomyelitis? Orthop Clin North Am 1991; 22: 467-71.

2. Campoccia D, Montanaro L, Arciola CR. The significance of infection related to orthopedic devices and issues of antibiotic resistance. Biomaterials 2006; 27 : 2331-9.

3. Chen CE, Ko JY, Fu TH, Wang CJ. Results of chronic osteomyelitis of the femur treated with hyperbaric oxygen: a preliminary report. Chang Gung Med J 2004; 27: 91-7.

4. Chen CE, Shih ST, Fu TH, Wang JW, Wang CJ. Hyperbaric oxygen therapy in the treatment of chronic refractory osteomyelitis: a preliminary report. Chang Gung Med J 2003; 26: 114-21.

5. Concia E, Prandini N, Massari L, et al. Osteomyelitis: clinical update for practical guidelines. Nucl Med Commun 2006; 27: 645-60.

6. Costerton JW, Stewart PS, Greenberg EP. Bacterial biofilms: a common cause of persistent infections. Science 1999; 284: 1318-22.

7. Cucarella C, Tormo MA, Knecht E, et al. Expression of the biofilm-associated protein interferes with host protein receptors of Staphylococcus aureus and alters the infective process. Infect Immun 2002; 70: 3180-6.

8. Darley ESR., Macgowan AP. Antibiotic treatment of Gram-positive bone and joint infections. J Antimicr Chem 2004; 53: 928-35.

9. Donlan RM. Biofilms and device-associated infections. Emerg Infect Dis 2001; 7: 277-81.

10. Gentry LO. Management of osteomyelitis. Int J Antimicrob Ag 1997; 9: 37-42.

11. Gitelis S, Brebach GT. The treatment of chronic osteomyelitis with a biodegradable antibiotic-impregnated implant. J Orthop Surg 2002; 10: 53-60.

12. Huang KC, Hsu WH, Peng KT, Huang TJ, Hsu RW. Hyperbaric oxygen therapy in orthopedic conditions: an evaluation of safety. J Traumatol 2006; 61: 913-7.

13. Lazzarini L, Lipsky BA, Mader JT. Antibiotic treatment of osteomyelitis: what have we learned from 30 years of clinical trials? Int J Infect Dis 2005; 9: 12738.

14. Lazzarini L, Mader JT, Calhoun JH. Osteomyelitis in long bones. J Bone Joint Surg Am 2004; 86: 2305-18.

15. Lentino JR. Prosthetic joint infections: bane of orthopedists, challenge for infectious disease specialists. Clin Infect Dis 2003; 36: 1157-61.

16. Lew DP, Waldvogel FA. Osteomyelitis Lancet. 2004; 364: 369-79.

17. Mast NH, Horwitz D. Osteomyelitis: a review of current literature and concepts. Operative Techniques in Orthopaedics (Treatment of Orthopaedic Infections) 2002; 12: 232-41.

18. Patzakis MJ, Zalavras CG. Chronic posttraumatic osteomyelitis and infected nonunion of the tibia: current management concepts. J Am Acad Orthop Surg 2005; 13: 417-27.

19. Prandini N, Lazzeri E, Rossi B, Erba P, Parisella MG, Signore A. Nuclear medicine imaging of bone infections. Nucl Med Commun 2006; 27: 633-44.

20. Salvana J, Rodner C, Browner BD, Livingston K, Schreiber J, Pesanti E. Chronic osteomyelitis: results obtained by an integrated team approach to management. Conn Med 2005; 69: 195-202.

21. Soriano A, Popescu D, Garcia S, et al. Usefulness of teicoplanin for preventing methicillin-resistant Staphylococcus aureus infections in orthopedic sur- 
gery. Eur J Clin Microbiol Infect Dis 2006; 25: 35-8.

22. Stein H, Lerner A. Advances in the treatment of chronic osteomyelitis. Curr Orthop 2001; 15: 451-6.

23. Wang J, Li F, Calhoun JH, Mader JT. The role and effectiveness of adjunctive hyperbaric oxygen therapy in the management of musculoskeletal disorders. J Postgrad Med 2002; 48: 226-31.

24. Zuluaga AF, Galvis W, Saldarriaga JG, Agudelo M, Salazar BE, Vesga O. Etiologic diagnosis of chronic osteomyelitis. Arch Intern Med 2006; 166: 95-100. 